DPROTEOMICS

\section{Getting the numbers right}

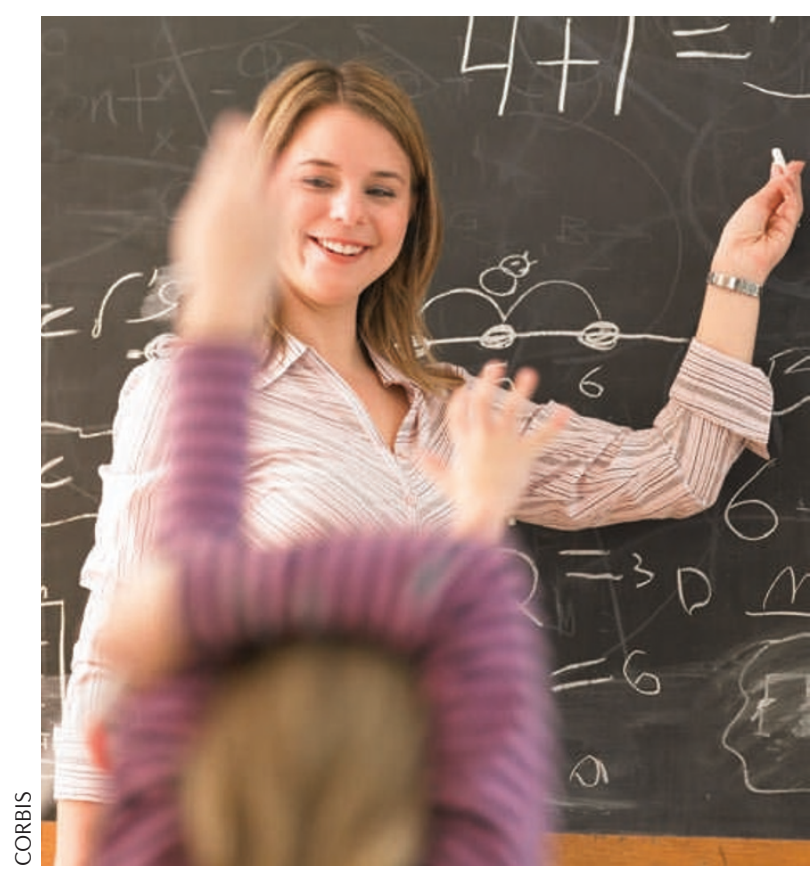

One of the main challenges in mass spectrometry (MS)-based methods is to achieve fast and precise quantitative analysis of any protein (or set of proteins) of interest in a proteome. According to two new studies by Ruedi Aebersold and colleagues this is now possible.

Malmström et al. developed an integrated MS strategy to estimate the cellular concentration of proteins for a large fraction of the proteome of the bacterial human pathogen Leptospira interrogans. The authors' strategy consisted of a three-step approach. First, they used highthroughput proteome sequencing by liquid chromatography-tandem MS to identify proteins in whole-cell protein extracts and to determine, for each identified protein, the three unique peptides with the highest signal intensity. Next, they determined the absolute amounts of the reference peptides using isotope labelling and selected reaction ion monitoring (SRM) MS to generate anchor points for the abundance calibration. Last, using the anchor points to calibrate the precursor ion intensities and spectral counts, they obtained absolute protein quantities for $51 \%$ of the predicted proteome of $L$. interrogans.

To validate the MS-derived data, Malmström et al. determined the amounts of selected proteins using an alternative method. They measured the dimensions of different $L$. interrogans structures (such as the flagellum) by cryo-electron tomography and, based on these measurements, calculated how many copies of the proteins known to constitute these structures were present for each cell. The results confirmed the accuracy of the MS-derived data. Notably, the new quantification method also enables the abundance of specific proteins to be compared between different samples. For example, following exposure of $L$. interrogans to an antibiotic, the authors found that the amounts of a few low-abundance proteins were highly increased, whereas the total cellular protein content remained constant.

Using an SRM-based proteomics approach, Picotti et al. detected 100 selected proteins from the total cellular proteins of Saccharomyces cerevisiae over a range of concentrations from over a million to fewer than 50 copies per cell. They detected proteins that have not been detectable before by other approaches, and showed that proteins can be detected at fewer than 10 copies per cell. Furthermore, the authors were able to quickly and reliably quantify 45 proteins that span a broad abundance range in the central carbon metabolism network of S. cerevisiae under different metabolic conditions. The comparison of the protein profiles to the corresponding transcript profiles provides new information on the timing and regulation of this protein network.

As the authors state: “...the SRM approach provides a simple, economical and fast way to explore the dynamics of cellular pathways, which will find broad applications in systems biology, but also in medical and pharmaceutical research.... So, proteomics studies can finally get the numbers right.

Francesca Cesari

ORIGINAL RESEARCH PAPERS Malmström, J. et al. Proteome-wide cellular protein concentrations of the human pathogen Leptospira interrogans. Nature 460, 762-765 (2009)| Picotti, P. et al. Full dynamic range proteome analysis of $S$. cerevisiae by targeted proteomics. Cell 6 Aug 2009 (doi:10.1016/j.cell.2009.05.051) FURTHER READING Gingras, A.-C. et al. Analysis of protein complexes using mass spectrometry. Nature Rev. Mol. Cell Biol. 8, 645-654 (2007) 